\title{
Monika Frommel Zaghafte Versuche einer Reform der sexuellen Gewaltdelikte
}

Der im Mai 1996 vom Bundestag beschlossene Gesetzesentwurf der Union zur Änderung des Sexualstrafrechts (Vergewaltigung in der Ehe) ist eigentlich ein Erfolg. Dennoch baben ihn weder die SPD noch die Grünen begrüßt, da er das umstrittene Widerspruchsrecht verletzter Ehefrauen enthält. Dieses kann, muß aber nicht, zur Umgehung des Reformziels führen. Im folgenden wird der Kontext der langwierigen Reformbemühungen gezeigt und eine möglichst effektive Umsetzung angeregt.

D. Red.

\section{Einleitung}

Das Gesetz zur Änderung des Sexualstrafrechts (1996) bestraft die eheliche Vergewaltigung als Verbrechen, erweitert den deliktspezifisch engen Gewaltbegriff in $\mathbb{S} 177,178 \mathrm{StGB}$ und erhöht die Strafdrohung, da der minderschwere Fall der Vergewaltigung mit der nicdrigen Strafdrohung von sechs Monaten gestrichen wird. Auf den ersten Blick ist dies ein Erfolg, dennoch haben weder die SPD noch die Grünen den Entwurf bcgrüßt, da er bei patriarchaler Nutzung Strafverfahren gegen verheiratete Täter faktisch unterbinden könnte. Das umstrittene Widerspruchsrecht mißhandelter Ehcfraucn kann, muß aber nicht diesen Elfekt haben. Es hat ihn, wenn keine Routinen entwickelt werden, betroffenen Frauen die Angst vor einem Strafverfahren zu nehmen. Das Widerspruchsrecht kann aber rein symbolisch blciben, wenn die Staatsanwaltschaften trotz crklärtem Widerspruch immer dann ein Strafverfahren einleiten, wenn dies im öffentlichen Interesse liegt. Die tatsächliche Umsetzung der Reform hängt also von der Interpretation des unbestimmten Begriffs des öffentlichen Interesses ab. Vergleichbares gilt für den erweiterten Gewaltbegriff. Das neu eingefügte Merkmal des "Ausnutzens« cincr hilflosen Lage kann gerichtlich ausgebaut werden oder bedeurungslos bleiben. Die Implementation von Reformgesetzen obliegt der dritten Gewalt. Jede kluge Gesetzgebung berücksichtigt dies. Im folgenden soll daher historisch und rechespolitisch der Hintergrund dieser zaghaften Reform gezeigt werden, um cine möglichst effektivc Umsctzung anzuregen.

Seit den $1980 e r$ Jahren ist informierten Beobachtern klar, daß das Sexualstrafrecht kein überzeugendes Konzept hat. Zwar strebte die Strafrechtsreform 1968-1975 an, das sexuelle Selbsrbestimmungsrecht abschließend und umfassend zu regeln, doch gelang dies nicht. Bci genauerem Hinsehen sind die Strafnormen lückenhaft und enthalten zahlreiche Wertungswidersprüche. Es fehlt ein Vergehenstarbestand, der den »Normalfall« sexueller Übergriffc erfaßt. Dic Verbrechenstatbestände der Vergewaltigung und sexuellen Nötigung sind im geltenden Recht an die hohe Schwelle des herkömmlich engen Gewaltbcgriffs gebunden, so daß es in der Praxis zu auffällig gehäuften Freisprüchen oder extrem niedrigen Strafen kam. Die Wertungswider- 
sprüche könnten durch eine problembervußte Rechtsrvissenschaft und eine entsprechend aufmerksame Rechtsprechung harmonisiert werden. Aber genau diese Erwartung wäre naiv und würde ignorieren, daß die traditioncll männlich besetzte Strafjustiz hierzu gerade nicht in der Lage war und dies auch heute bei gestiegenem Frauenanteil noch nicht ist. Dennoch hat die seit über zwanzig Jahrcn in regelmäßigen Abständen geäußerte Kritik und die geradezu notorisch schlechte Presse bei zahlreichen fragwürdigen Strafurteilen dic Strafverfolgungsbehörden nicht unbeeindruckt gelassen. Da aber Instanzgerichte immer nur einzelfallbezogene Auswege suchen können und überdies die Revisionsgerichte selbst diese zaghaften Versuche wieder unterbinden, hat sich das Sexualstrafrecht zu einer unübersichtlichen Materic entwickelt. Es gibt nicht nur erhebliche rcgionale Unterschiede, sondern auch für die Opfer zermürbende Differenzen zwischen Instanz- und Revisionsgerichten. Selbst BGH-Scnatc sind sich untereinander uneins, vermeiden aber gleichwohl, den gemeinsamen Großen Senat zur Klärung anzurufen. Wie so häufig bei rveltanschaulich umstrittenen Fragen reagieren die Kritisierten entweder diplomatisch oder stur, d. h. nach dem Motto: Seit wann läßt sich die dritte Gewalt durch externe Kritik beeindrucken. ' Aber langfristig wirkt extcrne Kritik, zumal wenn sic crkennbar berechtigt ist, eben doch. Seit einigen Jahren wird die Forderung nach einer Gesamtreform des Sexualstrafrechts nicht mehr als »feministischer Ruf nach härterem Strafrecht « abgewertet ${ }^{2}$, sondern aufgegriffen. Kompromisse werden sichtbar und realistisch, wie die Formulierung im Entwurf der Union zeigt. Offenbar kann sich dic Justiz auf Dauer nicht vorhalten lassen, die knappe Ressource Strafrecht evident ungleich zu nutzen: unangemessen restriktiv und opferbeschuldigend bei sexueller Gewalt und sexuellem Mißbrauch, unangemessen extensiv und unter präventiven Gesichtspunkten sinnlos bei den massenhaft auftretenden Eigentumsdelikten ${ }^{3}$, skandalös inhuman bei der noch immer üblichen Kriminalisierung abhängiger Drogenkonsumenten ${ }^{4}$, um nur einigc Bcispicle von unglcicher Nutzung des Strafrechts zu nennen.s

\section{Strafrechtliche und außerstrafrechtliche Interventionen gegen Gewalt in engen sozialen Beziehungen - eine erste Beschreibung des Problems}

Nach über zehn Jahren zermürbend langweiliger Dcbatten scheint Überdruß einen Kompromiß zu ermöglichen. Alle Parteien sind sich einig, daß Wort "außerehclich “ in $\$ \$ x>7, x>8 \mathrm{StGB}$ zu streichen und gesetzlich klarzustellen, daß schweres Unrecht nicht "minder schwer wiegt, wenn Täter und Opfer sich kennen oder verheiratet sind. ${ }^{6}$ Dies ist zweifellos ein Fortschritt. Eine zweite - noch umstrittene und leider

1 Auffallend unetnsichag reagiert etwa der 2. Senat des BGH, vgl. hierzu Beispicle bei Frommel, in: Neue Kriminalpolitik $3 / 1993$, S. 22 ff.

2 Vgl. noch dic Polemik von Sebastian Scheerer, KJ $3 / 1985$ und die Replik von Biggi Bender, KJ $4 / 1987$.

3 Ein Ungleichgewicht, das nicht abnimmt, sondern sich neuerdings sogar verscharft. Man denke nur an dic Hochstufung des Bandendiebstahls zum Verbrechen in $\$ 244$ a StGB, neu gefaßt durch das OrgKG, BTDrs. $12 / 989$, S. 25 .

4 Umfassend hicrzu Haffke, ZStW 1995, S. $761 \mathrm{ff}$.

s Mit dieser Begrundung betont der rechtspolitische Sprecher der Grunen die punktuelle Forderung nach intensiverer Strafverfolgung bei sexueller Gewalt trotz sciner insgesamt strafrechtsskeptischen Haltung, vgl. Volker Beck, ZRP 1995, 281 ff. (285).

6 Die Streichung des Merkmals maußerehelichs fuhrt nur dann zu einer Gleichbehandlung von sexueller Gewalt in und außerhalb enger personlicher Beziehungen, wenn auch der traditionell opferbeschuldigend konstruierte "minderschwere Fall der Vergewaltigung entfallt, da dic Gerichte und die KommentarLitcratur typischerweise das Tatunrecht unter- und die ${ }^{2}$ Tater-Opfer-Beziehunge überbewerten. Vgl. 
auch noch unbeantwortete - Frage ist, wie in der langen Zeit zwischen der Einleitung eines Strafverfahrens und dessen Abschluß der Opferschutz tatsächlich verbessert werden kann. Hier sind umfassende Konzepte gefragt, denn es genügt nicht, hohe Strafen zu fordern, es muß auch ein vor Ort abrufbares Hilfsangebot geben, und zwar flächendeckend. Leider gibt es nur erste Ansätze. Zwar haben sich in den letzten zwanzig Jahren Projektc und Arbeitskreise gebildet, die anstrcben, alle mit dem Problem der "Gewalt im sozialen Nahraum "bcfaßten und in Einzelfall auch zuständigen Institutionen zu vernetzen.? Aber diese Ansätze sind noch nicht zur altäglichen Routine geworden. Es macht ja keinen Sinn, ein Strafverfahren einzuleiten, wenn das Opfer danach noch mehr Repressalien fürchten muß.

Nicht nur Strafrecht, sondern auch Zivil- und Iamilienrecht sind gefragt. Bei gewalttätigen Ehemännern etwa ist zu prüfen, ob die Ehewohnung durch das Familiengericht der mißhandelten Frau zugewiesen werden soll, damit es zu einem vorläufigen Stillstand der Gewalt konmen kann. Erst dann sollte die Symbolik des Strafrechts genutzt werden, denn isoliert ist Strafrecht cine stumpfe Wafte. Nur cine Kombination von strafrechtlichen und außerstrafrechtlichen Reaktionen wirkt gegen Täter, die ihre Opfer gut kennen. Folglich muß es Anlaufstellen geben, die hilfesuchenden Frauen Hilfe und Beratung anbieten und ihnen Wege zeigen, wie sie aus Isolation und finanzieller Abhängigkeit herauskommen können. In den letzten Jahrzehnten haben sich zahlreiche Initiativen zur Verbesserung des Opferschutzes gebildet, Projekte der autonomen Frauenbewegung, aber auch solche, die einen halbstaatlichen Charakter haben. Selbst in konservativen Regionen gibt es mittlerweile entsprcchende Ansätze, wie z. B. das Passauer Projekt. ${ }^{8}$ Die mittlerweile gut institutionalisierten Notrufe reagieren cbenso wie die vereinzelt angcbotenen Interventionsprojekte auf die alles andere als neue Erkenntnis, daß Opfer gewalttätiger Beziehungen niedrig-schwellige Angebote brauchen, um aus einem Leben auszusteigen, das von Gewalterfahrung geprägt ist. Strafverfahren wirken nur, wenn cs auch außerstrafrechtliche Interventionen gibt. Umgekehrt grcifen Hilfsangebote oft nur, wenn es auch repressive Alternativen gibe und wenn diese auch tatsächlich genutzt werden. Die abolitionistische Ideologie der 1970er Jahre, »Hilfe statt Strafe«, ist mittlerweile einem systemischen Ansatz gewichen. Nicht der einzelne Täter, sondern soziale Faktoren der Normstabilisierung oder Destabilisierung stchen im Vordergrund. Das Ziel ist es, unterschiedliche Reaktionen zu kombinieren und dabei zu lernen, was die konkurricrenden Teilsysteme dessen, was man formellc soziale Kontrolle nennt, leisten können und wo ihre jeweiligen Grenzen sind. Blcibt zu klären, in welchem Verhältnis die beiden ganz unterschiedlich strukturierten Formen der Intervention zueinander stehen. 1994 (S. 276 ff., S. 300 ff.).

7 Zum sozialwissenschaftlichen Begriff der „Vernetzung*vgl.: M. Hege/G. Schwarz, Gewalt gegen Kinder. Zur Vernetzung sozialer Unterstutzungssysteme im Stadteeil, Munchen 1992, S. 112, 160 (Problem des Helferinnenegoismus). Grundlegend ferner: H.Keupp/B. Rohrle ( $\mathrm{Hg}_{\mathrm{g}}$ ), Soz.lale Netzwerke, Campus 1987.

8 Werner Beulke, Gewalt im sozialen Nahraum, MSchrKrım 1994, S. 360 ff. Das Passauer Modell ist in erster Linie ein Strafverfahren vermeidendes Modell. Aber seine Existenz zeigt, daß in allen Bundeslandern mehr oder weniger ausgepragt die Bereitschaft existuert, effektiveren Opferschutz zu erproben. Vgl. fur die Situation in der Bundesrepublık: Peter Wetzels, KFN Niedersachsen, Forschungsberichte Nr. 49, 1995. 


\section{Strafbarkeit der Vergewaltigung in der Ehe - ein erster Schritt zu einer Gesamtreform der strafrechtlichen Regelungen?}

In den letzten zehn Jahren scheiterte eine Gesamtreform des Sexualstrafrechts in erster Linie an der Weigerung der konservativen Regierungen, die eheliche Vergewaltigung nach $\oiint_{177} \mathrm{StGB}_{\mathrm{t}} \mathrm{u}$ bestrafen (als Verbrechen und nicht über den Umweg des besonders schweren Falls der Nötigung nach $\$ 240$ Abs. 1, 2. Alt. ${ }^{9}$ ). Es sieht so aus, als sei dicser Widcrstand überwunden. Immerhin finden sich noch Reste eines patriarchalen Sonderrechts beim sog. Widerspruchsrecht der Ehefrau $(\$ 177$ Abs. $s$ des Koalitionsentwurfs - vgl. die Synopse am Ende). Aber bevor voreilig spekulicrt werden soll, betrachten wir die Vorgeschichte und den Text des Koalitions-Entwurfs genauer.

Nimmt man die Begründung des Unionsentwurfs crnst, dann wird nun auch von der Koalition eingeräumt, daß sowohl die ungleiche Vcrfolgungspraxis als auch die unangemessenc Rechtsprechung nach einer Gesamtreform des Sexualstrafrechts verlangt. In ihrem 1995 eingebrachten Änderungs-Entwurf stellen CDU, CSU und FDP fest, daß der Schutz des sexuellen Selbstbestimmungsrechts lückenhaft ist. Die frühere Justizministerin Leutheusser-Schnarrenberger begründet den Entwurf mit den Worten:

"Mit der heutigen crsten Lesung des Gesetzentwurfs der Koalitionsfraktionen wird eine Reform des Sexualstrafrechts eingeleitet ...«. Der Entwurf selbst bestimmt als Ziel einen umfassenderen Schutz des sexuellen Selbstbestimmungsrechts: „Durch die gegenwärtige Fassung der $\int \mathbb{S} 177 \mathrm{ff}$. StGB ist das sexuelle Selbstbestimmungsrecht nicht genügend geschützt ... In Fällen, in denen weder Gewalt ausgeübt noch mit gegenwärtiger Gefahr für Leben und körperliche Unversehrtheit des Opfers gedroht wird und dieses die Tat aus Angst vor Körperverletzungs- und gar Tötungshandlungen des Täters über sich ergehen läßt, bestehen Strafbarkeitslücken « (Entwurf eines Strafrechtsänderungsgesetzes $\$ \int 177$ bis 179 , vom 27.9. 1995, BTDrs. $\left.1_{3} / 2463\right)$.

SPD und Grüne trauten zunächst diesem Bekenntnis nicht. Jedenfalls nutztc dic SPD die Situation nicht offensiv, sondern verhielt sich paradox, nennen wir es: traditionalistisch im gebremsten Reformwillen. Zwar ist es verdienstvoll, daß die SPD seit 1983 das Thema legislativ aktuell hält. Aber offenbar sprechen sich die frauenpolitischen und rechtspolitischen Gruppen nicht präzise genug ab. Die neue Situation hätte jedenfalls mehr Behutsamkeit verlange. Ohne auf den Unionsentwurf einzugehen, brachte die Fraktion in der 1 3. Legislaturperiode lediglich ihre früheren Versionen ohne qualitative Verbesserungen erneut ein, so daß der Koalitionsentwurf die Debarte bestimme.

Etwas anders ist die Situation bei den Grüncn. Frauenpolitik und Rechtspolitik sind mittlerweile gut koordiniert, aber beide Gruppen haben Probleme, sich auf eine Linie zu einigen. ${ }^{\circ}$ Offenbar ist es nicht so leicht, die beiden - in verschiedenen Szenen geführten - Diskurse zu einem politischen Konzcpt zusammenzuführen: den

$9 \$ 240$ Abs. t, 2. Alt. droht mit einer Mundeststrafe von 6 Monaten, d. h., wenn man davon absieht, daß die Notigung ein Vergehenstatbestand ist, derselben Mindeststrafe, die der umstrittene nminderschwere* Fall des Verbrechenstatbestandes der Vergewaltigung nach nocb geltendem Recbt z.Zt. ebenfalls vorschreibt.

10 Die gemeinsame Kompromißlinie zengt Volker Beck (Fn. 5), S. 28 s. Die hier verterene Losung versucht, feministusche Akzente und krimınalpolitısche Reformansatze so aufeinander abzustimmen, daß ein in sich stimmiges Konzept entsteht. 
feministischen auf der einen und den kriminalpolitischen auf der anderen Seite. Wegen dieser Mühen und des kurzatmigen realpolitischen Zwangs, das Aktuelle dem Langfristigen täglich opfern zu müssen, haben die Grünen auf einen eigenen konstruktiven Vorschlag verzichtet. Offenbar sind auch sie überrascht worden und waren auf eine kurzfristig angelegte pragmatische Reaktion nicht eingestellt.

Verständlich ist dicse langsame Reaktionsgeschwindigkeit von SPD und Grünen, da die notorisch patriarchale Haltung der Regierung sie in einer bequemen Fundamentalopposition belassen hatte, eine Haltung, die aber nicht mehr adäquat ist, seit sich pragmatische Verbesserungen abzeichnen.

\section{Historischer Rückblick}

Vor zehn Jahren, bei der ersten Anhörung des Rechtsausschusses am 26.6. 1986, war das Thema "Vergewaltigung in der Ehe" noch ein polarisierendes und für markige Sprüche selbstgefälliger Herren ausgesprochen anfälliges Thema. Aber auch damals sprachen sich vor dem Rechtsausschuß fast alle Sachverständigen für dic Streichung des Merkmals "außerehelich « im Vergewaltigungsparagraphen aus. Dennoch scheiterten damals selbst kleine Schritte in Richtung ciner Reform kläglich." Zehn Jahre später, am 6. 12. 1995, tagee der Rechtsausschuß erneut. Die noch zu klärenden Fragen sind mittlerweile pragmatischer Art und werden auch so behandelt. Keinc Spur von Geschlechtcrkampf, sondern eine Reform "von oben ", die so gut wie kein Interesse der Presse entfacht. ${ }^{12}$ Was vor eincm Jahrzehnt noch die Gemütcr aller bewegte, nämlich die strittige Frage der Mindeststrafe von zwei Jahren ohne eine Minderungsmöglichkeit über dic Annahme eines minder schweren Falls der Vergewaltigung (Antidiskriminierungsgesetz der Grünen $\times 984 / 1985$ ), wird nun von der Koalition mehr oder weniger als konsensfähig vorausgesetzt. Gestritten wird nur noch auf Nebenschauplätzen, über das im Koalitionsentwurf vorgesehene Widerspruchsrecht der Ehefrau (oder die sog. Versöhnungsklausel der SPD bei einer »eheähnlich « mit dem Beschuldigten zusammenlebenden Frau). Oder trügt der Schein?

Im folgenden sollen zwei Fragen untersucht worden. Wie ist der im Koalitionsentwurf ncu formulierte Tatbestand der sexuellen Nörigung/Vergewaltigung zu interpretieren (III. 3). Wie wirkt sich das vorgesehene Widcrspruchsrecht aus bzw. gibt es bessere Lösungen (IV.), die die praktischen Probleme der Betroffenen ernst nehmen, also niche in dem Sinne "symbolisch « sind, daß sie plakativ etwas normieren, das sclbst gutwillige Richter und Richterinnen nicht umsetzen können. Schließlich muß eine Reform die Situation der von sexueller Gewalt und Mißbrauch Betroffenen auch faktisch verbcssern (hierzu V.).

11 Monika Frommel, Das klagliche Ende der Reform der sexuellen Gewaltdelikce, ZRP 1988, S. 233 ff. Vgl. ferner die "Vorschlage zur Reform des Sexualstrafrechts $\propto$, in: Strafrecht - ultima ratio, Empfehlungen der Niedersàchsischen Kommission zur Reform des Strafrechts und des Strafverfahrensrechts, Peter-Alexis Albreche u. a. (Hrsg.), 1992, S. $47 \mathrm{ff.}$

12 In der Presse wurde uber den neu gefaßten Einheitstatbestand der sexuellen Notigung/Vergewaltigung fast nicht mehr debattiert, sondern nur noch uber den Streıt zwıschen Anhangern des Widerspruchsrechts und denen einer sog. Versobnungsklausel (Justızministerin und SPD-Fraktion) berichtet. Die Entwurfe der kleinen Parteien, etwa Stellungnahmen der Grunen blieben Zeıtungslesern unbekannt. Ausnahmen bestätigen lediglich diese Regel: informiert hatte uber den Streitstand der *zweiwochendienst Frauen und Politik:, Nr. 107/1995, Bonn, S. $10 \mathrm{ff}$. 


\section{3. Änderungsvorschlag der Koalition: der Einheitstatbestand der sexuellen Nötigung/Vergewaltigung}

Schon vor einem Jahrzehnt, der damals geplanten, aber 1988 gescheiterten Reform der sexuellen Gewaltdeliktc, hatte das BMJ einen Einhcitstatbestand der sexuellen Nötigung vorgeschlagen. Aber damals zielte die Abstufung in besonders schwere und minderschwere Fälle der sexuellen Nörigung auf eine Flexibilisierung der Strafzumessungspraxis. Mittlerweile hat das BMJ die damals geäußerte Kritik konstruktiv verarbeitet. Der Einheitstatbestand ermöglicht nun cine flexible, aber in Ergebnis akzeptable Abstufung der Schwere des jeweils verwirklichten Unrechts. Die bisher als Vergewaltigung strafbaren Tathandlungen sind unter Einbeziehung oraler und analer Praktiken als besonders schwerer Fall der sexuellen Nötigung (Vergewaltigung) gesetzlich neu definiert. Der minderschwere Fall der sexuellen Nötigung bezieht sich - schon aus der äußeren Systematik erkennbar - auf sexuelle Handlungen, die nicht mit einem Eindringen in den Körper (dann ist die Tat als Vergewaltigung definiert) verbunden sind. Der immer umstrittene, da zu opferbeschuldigenden Verteidigungsstrategien führende minderschwere Fall der Vergewaltigung ist abgeschafft.

Dies bedeuter, daß zumindest ein im Antidiskriminierungsgesetz ( $1984 / 1985$ ) der Grünen gefordertes Reformziel von der Koalition nach einem Jahrzehnt erfüllt worden ist: die Streichung des minderschweren Falls der Vergerwaltigung. Der neu geschaffene Einheitstatbestand geht insofern - paradoxerweise - weit über das hinaus, was die SPD-Entwürfe (vgl. Synopse) seit 1983 vorschlagen. Was den Gewaltbegriff betrifft, so wird dessen Erweiterung im Vorschlag der Grünen (gegen den Willen) nicht aufgegriffen. Aber der moderate SPD-Vorschlag: Erweiterung durch das Merkmal des »Ausnutzens einer hilflosen Lage" wird weitgehend übernommen. Bekanntlich hat die Rechtsprechung Probleme mit sich nicht wehrenden, da vor Angst wie "gelähmten " Fraucn - eine besonders häufige Konstellation. Opferbeschuldigende Freisprüche sollen in Zukunft vermieden werden durch das neu eingefügte Merkmal des "Ausnutzens ciner Lage, in der das Opfer dem umgehemmten Einfluß des Täters preisgegeben ist $\alpha$. Die theatralische Formulierung läßt sich sprachlich vereinfachen, etwa durch die Formulierung des SPD-Vorschlags. Die Folge der Reform könnte ein Rechtsprechungswandel sein. Gerichte hätten die Gelegenheit zum geordneten Rückzug. Sie können nun fallbezogen von den Ungereimtheiten abrücken, die sie in der Vergangenheit durch ihre restriktive Rechtsprechung (zum Gewaltbegriff, Vorsatz, zur fingierten Einwilligung und zu Irrtumskonstruktionen etc.) produziert haben.

Bei der Anhörung am 6. I 2. 1995 haben mehr oder weniger alle Sachverständigen der Konstruktion des Einhcitstatbestandes und der Erweitcrung des Tatbestandes zugestimmt. Diskutiert wurde lediglich, ob der minderschwere Fall der sexuellen Nötigung, Mindeststrafe: 6 Monate, auch für besonders schwere Fälle, d. h. Vergewaltigungen, gelte. Aber offenbar hatte der Sachverständige Dirk Helmken, von dem dieser Vorschlag stammt, den fast zehn Jahre alten, mittlerweile revidierten BMJEntwurf vor Augen, als er meintc ${ }^{13}$, eine Tat könne "vom besonders schweren Fall zum minder schweren heruntergeschleust werden $\alpha$. Wäre dies zutreffend, hätte die von der Koalition vorgeschlagene Reform nur eine symbolische Bedeutung, da dann eheliche Vergewaltigungen von der Rechtsprechung als "minderschwere« Fälle behandelt werden könnten. Vor dem Rechtsausschuß habe ich Helmken widersprochen, was ihn und andere zu keiner Replik veranlaßt hat. Offenbar hat der

I3 Seite 3 des dem Ausschuß zugeleiteten Gutachtens. 
Koalitionsentwurf den Weg des "Herunterschlcuscns" konstruktiv abgeschnitten. Dics bedeutet, daß faktisch die Strafdrohung bei jeder Form der Vergewaltigung erhöht worden ist (Mindeststrafe: 2 Jahre).

Beim besonders schweren Fall der sexucllen Nötigung, so dic leicht erkennbare Systematik des ncuen Einheitstatbestandes, sind Sexualpraktiken gemcint, dic mit einem "Eindringen in den Körper verbunden sind. Hierin wird die besondere Schwere der sexuellen Handlung bei einer Vergewaltigung gesehen. Sollte ein Strafgericht nun in einem Einzelfall im Wege der Gesamtbetrachtung, die bei Regelbeispielen ausnahmsweise zulässig ist, den »besonders schweren Fall « trotz Eindringens in den Körper wegen sog. Gegenindikatoren (etwa das Vorliegen des $\ 21$ StGB) verneinen, muß es auf den Grundtatbestand zurückgreifen (Mindeststrafe: ein Jahr). "Herunterschleusen « auf den minder schweren Fall der sexuellen Nötigung (sechs Monate Mindeststrafe) kann es nicht mchr, da die minder schweren Fälle für weniger gravierende sexuelle Handlungen (etwa gewaltsames Busengrapschen) reserviert sind. Damit wird die Schwere des Unrechts an der Intcnsität der sexuellen Beeinträchtigung gemessen, was angemessen ist.

\section{Noch reformbedürftig: $\int 179 S t G B$}

Nicht bedacht hat der Koalitionscntwurf, daß \$ i 79 StGB in der vorgelegten Fassung leer läuft, da das "Ausnutzen " einer hilflosen Lage bei "Widerstandsunfähigkeit « regelmäßig zu bejahen ist, so daß nach der Novellierung hierfür der Verbrcchensund nicht der Vergehenstatbestand des \ I79 StGB einschlägig ist. Sinnvoll wäre cinc Neufassung (Synopse: Vorschlag Frommel). $\$ 179$ StGB sollte als Auffangtatbestand konstruiert und dic veraltetc und überdies Behinderte diskriminiercnde Definition der Widerstandsunfähigkeit vermieden werden. Sinnvoll wäre der Begriff der eingeschränkten Widerstandsfähigkeit. So gefaßt würde der Vergehenstatbestand des $\int_{179} \mathrm{StGB}$ Grenzfälle der Vergewaltigung (Grenze zum Ausnutzen einer hilflosen Lagc) abdecken und die häufigen Mißbrauchsfälle ohne grobe Gewalt crfassen. Dic Gründe für die im ncuen Gesetz nicht übernommene, aber hier vorgeschlagene Neuformulierung sind folgende: Der Anwendungsbereich von $\$ 179$ StGB des geltenden Rechts ist sehr begrenzt und wird von der Rechtsprechung überdies sehr eng ausgelegt. Also sollte $\ 179$ StGB erweitert werden. Dies würde Strafbarkeitslücken in den Fällen beseitigen, in denen das Opfer aus Angst und eincr dadurch hervorgerufenen körperlichen und psychischen Lähmung eine wirkungswolle körperliche Gegenwehr unterläßt, die Schwelle zum "Ausnutzen einer hilflosen Lage« (Verbrechenstatbestand) aber noch nicht erreicht ist.

Die hier vorgeschlagene Neufassung des $₫ 1_{79}$ StGB würde außerdem die Diskriminierung Bchinderter durch die unangemessene Beschränkung auf einc »biologisch «, d.h. nicht psychisch (Angst) bedingte Widerstandsunfähigkeit im geltenden Recht beseitigen. Aus diescm Grund wird nicht mehr an "Widerstandsunfähigkeit « angeknüpft, sondern an eine "eingeschränkte Widerstandsfähigkeit火. 
Schlicßlich sollte auch bei der Zuständigkeitsregelung nach dem GVG der seit 1987 (Opferschutzgesetz) geforderte, aber bislang unterbliebcne Opferschutz umgesetzt wcrden. Die sog. Rechtspflege-Entlastungsgesetze der letzten Jahre haben dazu geführt, daß bis zu einer zu erwartenden Strafe von vier Jahren die Amtsgerichte und damit zwei Tatsacheninstanzen zuständig sind. Diese Regclung widerspricht fundamental dem Prinzip eines angemessenen Opferschutzes, da sie Opter zwingt, zwci Tatsacheninstanzen durchzustehen.

$\$ 74$ Abs. 2 Nr. $1-3$ GVG ist dahingehend zu ändern, daß die Strafkammern beim Landgericht in erster Instanz bei Straltaten nach $\$ \mathbb{S}$ i $76-179$ StGB zuständig sind. Die ansonsten sinnvolle und wünschenswerte Entlastung des BGH (als Revisionsinstanz) hat gegenüber dem insoweit vorrangigen Opferschutz (Vermeidung ciner zweiten, aufwendigen und das Opfer belastenden Tatsacheninstanz) zurückzutreten.

\section{Sonderregelungen bei Beziebungstaten? Widerspruchsrecht, Versöhnungsklausel oder Verzicht auf jede Sonderregelung? Die Varianten der vorgelegten Entwürfe.}

\section{Die Flucht in die Diversion}

$\$ 177$ Abs. 5 StGB des Koalitionsentwurfs formulicrt das umstrittene Widerspruchsrecht einer vergewaltigten Ehefrau, $\ x_{77}$ Abs. 4 StGB des SPD-Entwurfs die mittlerweile von Frauenpolitikerinnen zurückgenommene Versöhnungsklausel (Ulla Schmidr, dpa vom 18. 1. 1996). Der F.ntwurf der Abgeordneten Schenk verzichtet auf jede Sonderregelung, während Bündnis 9o/Die Grünen die Einführung einer Vollstreckungsklauscl ${ }^{1+}$ prüfen. Was spricht für eine wie auch immer geartete Sonderregelung bei der ehelichen Vergewaltigung?

Stellt man die Frage so, ist die Antwort leicht. Es gibr keine spezifisch strafrechtlichen Probleme bei Beziehungstaten. Die praktisclıen Probleme, die es gibt, können besser durch nichtstrafrechtliche Interventionen gelöst werden. Dennoch gibt es gute Gründc, sich zu fragen, was passiert, wenn auf jede Sonderrcgelung verzichtet würde.

Der neu gefaßte Einheitstatbcstand hat nicht nur für eheliche Vergewaltigungen, sondern ganz allgemein den Tatbestand des $₫ 177$ StGB erweitert und die Mindeststrafe für Vergewaltigungen auf 2 Jahre angehoben. Wer will, daß die Gerichtc diesc Gesetzesänderung 7.um Anlaß nehmen für cine umfassende Änderung der Rechtsprechung, muß bedenken, daß der Wegfall des "minderschweren « Falls die Strafzumessung auf eine Reaktion einengt: eine nicht mehr zur Bewährung aussetzungsfähige Freiheitsstrafe. Illusionär also zu glauben, daß Gerichte das neue Gesetz konsequent umsetzen und den gesetzlich erweiterten Gewaltbegriff durch eine exklusive Auslcgung implementieren, wenn der Preis für diese Rechrstreue die Bindung an eine Mindeststrafe von zwci Jahren ist.

Strafgerichte wissen, daß Strafvollzug die problematischste Reaktion ist, die das

14 Ein entsprechendes Gutachten wurde von mir um Oktober i99s erstellt. Men Vorschlag wird am Ende dieses Artikels kurz begrundet. Die Grunen konnten sich aber zu einem eigenen Gesetzentwurf nicht durchringen. 
Strafgesetz kennt. Wenn sic also in Einzelfällen das Ergebnis "Gefängnisstrafe« vermeiden wollen, aus welchen Gründen auch immer, dann werden sie dies auch tun. Die Reform ist zum Scheitern verurteilt. Das neu geschaffene Recht wird nicht inplementiert. Schauen wir uns die nahe liegenden Schleichwege an: Gcrichte können weiterhin hohe Anforderungen an die Begriffe »Gewalt", "Drohung mit gegenwärtiger Gefahr für Leib oder Lcben« oder »Ausnutzen einer hilflosen L.age» stellen. Dicsen Effekt kann niemand wollen, der eine Reform anstrebt: die Erweiterung des Tatbestandes, um unangemessene Lücken zu schließen. Gerichte können ferner großzügig von der Möglichkeit der Strafminderung, etra bei Tätern, die Alkohol getrunken haben, Gebrauch machen. Auch dics ist nicht wünschenswert, da dieser Trend ohnehin seit Jahren feststellbar und mittlerweile ein Ärgernis ist. Alkoholgenuß ist lcicht meßbar und führt mit einem gewissen Formalismus zu Freispruch oder Strafmilderung. Kompliziertere Beeinträchtigungen werden hingegen strukturell von Gerichten ignoriert. Das Ergebnis ist cin Freibrief für Betrunkene und Angctrunkenc. Ein weiterer Weg liegt in der Ausnutzung der Rcgelbeispieltechnik. Gerichte können die Konstruktion des Einheitstatbestandes ausbauen und in ciner Vielzahl von Fällen den besonders schweren Fall verneinen, um zum Grundtatbestand zurückzukehren. Wer also nicht symbolische Gcsctz.c, sondern einen Anstoß zu einer grundlegenden Rechtsprechungsändcrung geben will, muß bei der Wahl der Rechtsfolgen einen sinnvollen Spielraum lassen, d. h. Möglichkeiten zur Vermeidung einer Freiheitsstrafe eröffnen.

\section{Erweiterung der Strafaussetzung zur Bewährung?}

Nahelicgend wäre es, angesichts der durch die Zweijahresfrist in $₫ 56 \mathrm{StGB}$ geschaffenen Zwangslage, dort anzusetzen und die Frist für eine Strafaussetzung zur Bewährung zu crweitcrn, also die Grenze von 2 Jahren nach oben zu verschieben. Dieser Vorschlag ist bereits $1992^{15}$ gemacht worden, aber damals auf berechtigte Kritik gestoßen. Die Strafaussetzung zur Bewährung hat sich in den letzten Jahrzehnten zu einer eigenen Strafart entwickelt, deren Sinn es ist, bei der überaus häufigen mittleren Kriminalität, d. h. nicht bei Gewaltkriminalität, zu vollstreckendc Freihcitsstrafen großflächig zu vermeiden. Die Anforderungen an die sog. positive Prognose sind sehr gering und die Rechrsprechung zum Widerruf einer Strafaussetzung ist mittlerweile so liberalisiert und gefestigt, daß in Zukunft auch die Quote der Widerrufe wohl sinken wird. ${ }^{16}$ Eine Veränderung der Fristen in $\$ s 6 \mathrm{StGB}$ würde diesen Prozeß mehr stören als fördern. Außerdem sind kontraproduktive Effekte zu befürchten. Die Zweijahresfrist diszipliniert die Strafzumessung. Daher sollte in einem so sensiblen Bereich nicht ohne Not experimentiert werden.

Wer also eine Mindeststrafe von mehr als 2 Jahren vorschlägt, muß sich Gedanken machen, wie diese Strafdrohung umgesetzt werden soll, ohne-dies ist eine Kritik in Richtung der notorischen Rufer nach Strafverschärfung in anderen Deliktsbereichen - nicht undifferenziert eine Verschärfung der Strafzumessung in Gang zu setzen, wie wir sie im Rahmen der Inneren-Sicherheits-Debatten beobachten konnten. Im Drogenstrafrecht und bei Eigentumsdelikten hat man immer wieder mit populistischen Argumenten die Mindeststrafe angehoben und dabei als informierter Beobachter auf

is Vgl. die Empfehlung der Niedersachsischen Kommission zur Reform des Stratrechts und des Strafverfahrensrechis (Fn. 11)

16 Zum Stand der Debatte vgl. Horn, Systematischer Kommentar 1992, \$56i., Rdnr. 8. 
Strafmilderungsmöglichkeiten und Strafaussetzungen zur Bewährung spekuliert.

Doch hat man in Endeffekt in einer Vielzahl von Fällen die Vollstreckung von sehr langen Freiheitsstrafen geerntet. Hohe Mindeststrafen wirken nicht generalpräventiv, sondern heben mit einer gewissen Zwangsläufigkeit das gesamte Strafenniveau an. Man sollre daher genau überlegen, ob cin Delikt tatsächlich mit einer Mindeststrafe von 2 Jahren bedroht werden soll. Entschließr man sich dennoch, bei der Vergewaltigung mit guten Gründen, dann solltc zumindest überlegt werden, wie man die Folgeprobleme bei der Vollstreckung mildern kann.

\section{Diskussion der Entwürfe}

Bündnis 90/Die Grünen suchen cinen konstruktiven Weg aus dem Dilemma im Sanktionenrecht (Vollstreckungslösung, vgl. hierzu V.), während der Entwurf der Abgeordncten Schenk (Synopse) auf jede Sonderregclung mit dem Argument verzichtet, daß nicht ausgerechnet bei dem Thema »Gewalt gegen Frauen neue Wege erprobt werden sollcn. Wie so oft, ist diese fundamentalistische Haltung nachvollziehbar, aber letztlich wenig effektiv, da Frauenpolitik und Kriminalpolitik gegeneinander ausgespielt werden. Auch die Haltung der SPD ist nicht mehr ganz klar nach den letzten dpa-Meldungen. Bislang hat auch sie sich in Formen der Diversion geflüchtet (Versöhnungsvorbehalt) und die Einleitung eines Verfahrens zur Disposition der Staatsanwaltschaft gestellt. Sie hat diesen Weg nur nicht offengelegt. Im SPD-Entwurf steht, das Gericht könne von Strafe absehen, wenn ... Wenn aber das Gericht von Strafe absehen kann, dann kann auch die Staatsanwaltschaft das Verfahren einstellen. Man hofftc also, Staatsanwaltschaften könnten bei Gurvaltbezichungen erfolgrcich ohne Einleitung eines Verfahrens intervenieren, weil sie dem 'lätcr mit einem Verfahren drohen können. Leider ist dies ein frommer Wunsch. Er erklärt auch das Widerspruchsrecht im Koalitionsentwurf. Auch dort meint man, die Frau solle das Drohpotential des Strafrechts nutzen, um eine freiwillige Umkehr des Täters zu »erzwingen «. Ich fürchte, beidc Scheinlösungen unterschätzen die Zwanghaftigkeit des Täterverhaltens und die Verstrickung von Täter und Opfer. Wenn sich nach heftigen Kämpfen endlich einmal ein Beziehungs-Opfer entschlossen hat, eine Strafanzeige zu stellen, sollte die Staatsanwaltschaft diese auch zügig bearbeiten und nicht erneut mit Täter und Vertcidigung verhandeln. Zu empfehlen ist, mit anderen zuständigen Institutionen zusammenzuarbeiten, die außerstrafrechtlich intervenieren bzw. Hilfsangebote machen können. Aber das Strafverfahren sollte nicht mehr von intcrnen Absprachen mit dem Beschuldigten abhängig gemacht werden. Zu klären ist, wie in der Zwischenzeit der Opferschutz verbessert werden kann (Erleichterung der Zuweisung der Ehewohnung etc.). Aber dic abstrakte Hoffnung auf das Drohpotential des Strafrechts, ohne die konkrete Furcht vor einem Verfahren, erscheint mir wenig realistisch. Potentielle Täter wissen ebenso wie potentielle Opfer, daß die hilflosen Hclfer sich selbst durch ihre Furcht vor Strafverfahren handlungsunfähig machen. Opfer resignieren und Täter lernen, daß sie auf wenig Gegenmacht stoßen.

Kritikwürdig ist also in erster Linie der Effekt, den Diversionsregeln haben. Sie verhandeln über die Einleitung eines Strafverfahrens. Signalisiert wird dadurch, daß eine repressive Intervention noch nicht unmittelbar bevorsteht. Dieses Zögern verschärft die Situation. Täter, die an Gewalt gewöhnt sind, können nicht frciwillig aufhören. Sie reagieren nur auf heteronomen Zwang. Erstaunlich, daß solche Lösun- 
gen dennoch immer wicder vorgeschlagen werden. Sie sind psychologisch falsch und juristisch schwer begründbar. Vergewaltigung ist ein Verbrechen. Es gilt das Legalitätsprinzip. Dogmatisch sind aber Diversionsregeln cine systcmfremde Einschränkung des Legalitätsprinzips. Nun könnte man sich über systematische Bedenken hinwegsetzen, wenn dic damit eröffneten Dispositionen angemessen wären. Aber dies sind sie nicht. Auch das von einigen Sachverständigen der CDU/CSU formuliertc Prinzip der „Opferautonomie «̈̈bcrzeugt nur auf den ersten Blick. Leider erweist es sich bei genauerer Kenntnis der Dynamik von Gewaltbeziehungen als eine Art "Juristenpsychologic «. Opfer von langjährigen Gewaltbezichungen sind besonders schlecht in der Lage, aus den $Z$ wängen dieser Art von Beziehung auszubrechen, sonst würden sich Übergriffe dieser Art nicht wiederholen und steigcrn. Dic Erprobung des Prinzips der "Opferautonomie* ist also bei dieser Fallgruppe nicht zu cmpfehlen.

Es sprechen aber noch andere Argumente gegen die vorgeschlagenen DiversionsRegeln. Es ist bekannt, daß bei Vergewaltigung (innerhalb und außerhalb von Beziehungen) die Dunkelziffer sehr hoch ist. Die Anzeigcbereitschaft ist trotz öffentlicher Aufmerksamkeit für das Thema in den letzten Jahren nicht gestiegen, obgleich dies zu erwarten war. Der Grund ist offenbar der, daß die Erwartungen der Opfer systematisch cnttäuscht worden sind, weil die Verfahren nach wie vor als unzumutbar und übermäßig belastend empfunden werden. Kriminalpolitisch sollte daher nicht die Einleitung von Strafverfahren vermieden, sondern die Opferautonomie im Verfahren gestärkt werden.

Ein dritter Einwand gegen das Widerspruchsrecht ist eher traditioneller Art. Es ist zu vermuten, daß der lange und zähe Widerstand gegen eine Reform der $\$ \$ 177$, I 78 $\mathrm{StGB}$ im Widerspruchsrecht seinen letzten Ausdruck gefunden hat. Es ist cin Relikt des alten patriarchalen Denkens, wonach die Ehe einc Beziehung ist, in der Sonderrcgclungen gelten müssen. Vertreter dieser Ansicht glauben offenbar, mit dem Widerspruchsrecht derartige Verfahren weitgehend vermeiden zu können. Zwar ist diesc Hoffnung trügerisch, weil dort, wo Sonderstaatsanwaltschaften tätig sind, täglich Erfahrung gesammelt wird, dic Ideologien auflösen wird. Erweist sich, daß viele Zeuginnen häufig nur unter Druck ihr Widerspruchsrccht betätigen, wird die Einleitung eines Verfahrens im öffentlichen Interesse die Regel. Wir befinden uns dann in der Schwierigkeit, wie wir mit dem Problem umgchen, daß die hohe Mindeststrafe den Spielraum der Gerichte bei der Wahl eincr angemessenen Strafe einengt.

\section{Neue Wege im Sanktionenrecht:}

\section{Intervention durch Auflagen und Weisungen?}

In der rechtspolitischen Diskussion wird in unterschiedlichsten Zusammenhängen die Frage erörtert, ob geeignete Weisungen und Auflagen, auch die Eimbeziehung justizexterner Interventionsprogramme - im Schatten strafrechtlicher Drohungen geeignet sein könnten, das Verhalten gewalttätiger Männer positiv zu beeinflussen. Gesucht wird nach einer Verbesserung des Opferschutzes jenseits strafrechtlicher Maßnahmen. Beobachtet man aber die Diskussion genaucr, fällt auf, daß die juristische und die therapeutische Berufsgruppe jeweils der anderen den "schwarzen Peter" zuweist. TherapeutInnen crwarten vom Strafrecht, cs möge den Kreislauf der Gervalt durchbrechen, bisweilen wird hinzugefügt: "ohne wenn und aber «, was eine gewisse 
Strafrechtsgläubigkeit verrät. Im strafrechtlichen Diskurs hingegen wird die Hoffnung geäußcrt, dic therapeutischen Berufe verfügten über ein Wissen und über geheimnisvolle Techniken, die der vergleichsweise groben strafrechtlichen Repression überlegen scicn. Die Formeln sind bekannt. Sie tauchen nicht nur bei "Gcwalt «, sondern auch bei "Jugendkriminalität " odcr "Drogenabhängigkeit « auf: "Hilfe und Strafe«, »Hilfe staut Strafc«. Sie prägen die Geschichte des spezialpräventiven Strafrechts seit der Jahrhundertwende.

Gegen eine zu enge Verzahnung beider Reaktionsformen spricht mittlerweile dic Erfahrung aus den Rechtsgebieten, in denen diese schon seit Jahrzehnten versucht wird: dem Jugendstrafrecht. Wir wissen mittlerweile aus der langen Geschichte der Vernetzung von Hilfe und Strafe im Jugendstrafrecht, daß eine zu enge und zu direkte Verknüpfung den Spiclraum bcider Teilsysteme der formellen sozialen Kontrolle einengt und die jeweilige Professionalität beider eher untergräbt statt fördert. Dies wird auch in der sozialwissenschaftlichen Litcratur thematisiert (vgl. Fn. 7). Das Subsystem »Hilfe« läßt sich nicht über reaktionsgenauere Auflagen und Weisungen steuern. Außerstrafrechtliche Interventionen sind auf die Kooperation mit dem Betroffenen angewiesen. Kommt sie nicht zustandc, dann scheitert das Hilfesystem und der Fall kommt zu den Strafverfolgungsbehörden zurück. Strafrecht ist gefragt, weil das andere System auch keine bessere Lösung findet. Fein intervenieren kann aber auch das Strafrecht nicht, weil es nur auf die simple Alternative reagieren kann, ob der Beschuldigte/Angeklagte/Verurteilte rückfällig geworden ist oder nicht. Die logische und praktische Folge ist die, daß es bei Rückfälligen nicht mchr abgestuft intervenieren und schon gar nicht ihr Verhalten positiv beeinflussen kann.

Dennoch sind in zahlreichen Deliktsbereichen die Leistungen einer auf Diversion und Haftvermeidung angelegten Kriminalpolitik bedeutsam. Im Bagatellbereich kann ein Strafverfahren unterbleiben (Einstellung nach Opportunitätsgrundsätzen), eine aussetzungsfähige Freihcitsstrafe kann zur Bewährung ausgesetzt werden, wenn zu erwarten ist, daß einschlägige Straftaten in Zukunft nicht mchr vorkommen. Zur Zeit unlösbare Probleme entstehen im wesentlichen nur bei langen Freiheitsstrafen. Bei ciner Verurteilung zu mehr als 2 Jahren Freihcitsstrafe enthält das Sanktionenrecht eine Lücke. Historisch sollte nämlich hier die Grenze der Haftvermeidung gezogen werden. Aber einleuchtend ist diese Grenze nicht. Im folgenden soll daher diese Lücke durch einen konstruktiven Vorschlag geschlossen werden. Unterstellt wird dabei, daß das moderne strafrechtliche Reaktionssystem mittlerweile so differenziert ist, daß in jeder Phase des Verfahrens die Abgabe eines Falls an außerstrafrechtliche Institutionen (oder Interventionsprojekte) möglich sein sollte. $\mathrm{Da}$ es gesetzlich nicht vorgesehen ist, daß ein zu einer Freiheitsstrafe verurteilter Täter die Strafe nicht antreten muß, sollte das Sanktionenrecht ergänzt werden.

\section{Aussetzung der Vollstreckung einer Freiheitsstrafe (Vollstreckungsklausel)}

Im Oktober r 995 haben die Grünen folgenden Vorschlag prüfen lassen. Wie könnte eine neu einzufügende Vorschrift im Allgemeinen Teil aussehen (etwa im Anschluß an die Regeln der Strafaussetzung zur Bewährung), nach der Verurteilungen zu einer nicht mehr aussetzungstähigen Freiheitsstrafe von mehr als 2 Jahren nicht vollstreckt werden müssen. Alternativ sollte geprüft werden, ob diese Klausel auf Sexualstraftaten begrenzt wcrden solle (am Ende des I3. Abschnitts, etwa als $\$ 184$ d StGB, 
einzufügen). Bei der Frage, welche Tätergruppe in Betracht kommt, ging ich in meiner Antwort von folgenden Überlegungen aus. Alkoholtäter werden durch das eingespielte System der Strafmilderung nach $\${ }_{21}$ StGB privilegiert. Sie bedürfen also keiner Sondervorschrift. Hingegen wird die spezifische Situation bei Konflikttaten an keiner Stelle berücksichtigt. Gerichte können es bei der Strafzumessung tun, aber nur dann, wenn sic Spielräume für Haftvermeidung haben. Aus diesem Grund bictet es sich an, die Vollstreckungsklausel an den unbestimmten Rechtsbegriff einer Konflikttat zu knüpfen, um diese Fallgruppen differenzierter behandeln zu können.

Formulierungsvorschlag eines neu einzufügenden $\int 56$ a n.F. (bzw. $184 d$ ) StGB:

(1) Ist jemand wegen einer Straftat

$(\$ 184 \mathrm{~d}$ : gegen die sexuelle Selbstbestimmung)

zu einer Freiheitsstrafe von nicht mehr als vier Jahren verurteilt worden und ergibt sich aus den Urteilsgründen oder steht sonst fest, daß er die Tat aufgrund ciner cinmaligen Konfliktsituation begangen hat, kann die Vollstreckungsbehörde mit $\mathrm{Zu}$ stimmung des Gerichts des ersten Rechtszuges die Vollstreckung der Strafe oder des Strafrestes für längstens zwei Jahre zurückstellen, wenn zu erwarten ist, daß künftig

$(\$$ I 84 d: das sexuelle Selbstbestimmungsrecht der verletzten und anderer Personen gewahrt bleibr)

vergleichbare Rechtsgutsverletzungen unterbleiben.

(II) Die Vollstreckungsbehörde widerruft die vorläufige Zurückstellung der Vollstreckung, wenn der Verurteilte

( $\$$ I 84 d: erneut das sexuelle Selbstbestimmungsrecht anderer Personen verletzt) erneut eine einschlägige Straftat begeht.

(III) Hat die Vollstreckungsbehörde die vorläufige Zurückstellung widerrufen, so ist sie befugt, zur Vollstreckung der Freiheitsstrafe cinen Haftbefehl zu erlassen. Gegen den Widerruf kann die Entscheidung des Gerichts des ersten Rechtszuges herbeigeführt werden. Der Fortgang der Vollstreckung wird durch die Aurufung des Gerichts nicht gehemmt. $\$_{4} 62$ StPO gilt entsprechend.

(IV) Ist dic Vollstreckung vorläufig zurückgestellt und nicht widerrufen worden, kann das Gericht unter den Voraussetzungen des $₫ 57$ StGB die Vollstreckung aussetzen.

Begründung:

Der Vorteil einer solchen Lösung wäre, daß es in allen Fällen einer erwiesencn sexuellen Nötigung/Vergewaltigung zu einer Verurtcilung kommt. Die Tat wird geahndet. Die Staatsanwaltschaft als Anklagebehörde bleibt an das Legalitätsprinzip gebunden. Es gibt keine systemwidrige Einschränkung dieses Prinzips bei einem Verbrechenstatbestand. Dennoch kann in geeigneten Fällen eine spezialpräventiv sinnlose Vollstreckung einer Freiheitsstrafe, die im Interesse des konkreten Opferschutzes nicht notwendig ist, unterbleiben.

Systematisch handelt es sich nicht um Diversion, sondern um Vollstreckungsrecht. Sie würde erst dann cingreifen, wenn eine Bewährungsstrafe nicht mehr möglich ist (Verurteilung von mehr als 2 Jahren). Für begründete Einzelfälle könnte dann von der Vollstreckung der Freihcitsstrafe ausnahmsweise abgesehen werden, wenn der Verurteilte Auflagen erfüllt, die eine positive Prognose rechtfertigen. Welche Auflagen geeignet sind, läßt sich nicht abstrakt am grünen Tisch festlegen. Entscheidend ist die Erwartung, daß es zu keiner Gewalthandlung mehr kommen wird. Praktisch wird dies in Fällen, in denen der Mann die Ehewohnung bereits verlassen hat, seinen Pflichten nachkommt und das Opfer stabilisiert worden ist, so daß es sich gegen Wiederholungen früherer Muster wehren kann. Derartiges kann kein Interventions- 
programm zur Auflage im strengen Sinne machen. Aber wenn diese Bedingungen dennoch eingehalten werden, wärc dic Vollstreckung einer Strafe mehr oder weniger sinnlose Vergeltung. Die Vollstreckungslösung ist daher insbesondere sinnvoll in Fällen, in denen der Gewaltkreislauf bereits durchbrochen ist.

Zuständig zur Überwachung der die Vollstreckung hemmenden Auflagen wäre die Gerichtshilfe bzw. die damit beauftragte Bewährungshilfe (da im Vollstreckungsverfahren die Staatsanwaltschaft zuständig ist).

Die Voraussetzung einer Konflikttat ist relativ leicht präzisierbar. Das geltende Recht sieht bislang privilegierende Sonderrcgelungen nur bei vermindert schuldfähigen Tätern vor. In den Genuß dieser Sonderregelung kommen etwa alkoholisierte Täter. Auch in Zukunft dürfte daher in der Praxis bei vermindert zurechnungsfähigen Verurteilten die Bewährungslösung von Bedeutung sein. Keine Berücksichtigung finden aber im geltenden Recht Konfliktsituationen. Diese können etwa (müssen es keincswegs) bei sexuellen Nötigungen und Vergewaltigungen in krisenhaften Beziehungen von Bedeutung sein. Kommt es zu einer Trennung und Auflösung der gemeinsamen Wohnung, könnte dies - neben anderen Auflagen - eine positive Prognose rechtfertigen. Daher wird im hier vorgeschlagenen Entwurf darauf abgestellt, ob der Täter die Tat aufgrund ciner einmaligen Konfliktsituation begangen hat. Der Grund für diese Auswahl ist der, daß bei Taten dieser Art typischerweise die $\$ \$ 20$, 21 StGB nicht greifen, so daß es kriminalpolitisch gut vertretbar ist, wegen des Tatunrechts eine harte Strafe zu verhängen, aber dennoch eine allgemein formulierte Ausnahmevorschrift für einmalige Konflikttaten vorzusehen.

Stellt sich die Frage, wie eine solche Vorschrift wirken würde. Parallele Erfahrungen, die aber nur unter Vorbehalt übertragen werden können, gibt es mit $\$ 3$ s BtMG. Im Gegensatz zu der hier vorgeschlagenen Lösung gilt $\$ 35$ BrMG nur bei Verurteilungen bis zur magischen Zweijahresfrist. ${ }^{17}$ Die bei Drogenabhängigen häufige Therapieweisung nach $\$ 56 \mathrm{StGB}$ (mit Einverständnis des Verurteilten) und die sehr komplizierte Vollstreckungslösung überschnciden sich also. 1982/1983, also kurz nach der Anhebung zahlrcicher Mindeststrafen im BtMG und der Einführung des $\$ 35 \mathrm{BtMG}$ (unter der falschen Flagge: "Therapie statt Strafe «), stieg das Strafniveau erheblich. Es wurden nach 1982 insgesamt mehr Freiheitsstrafen verhängt als vor der Strafschärfung. Die Verelendung der Drogenabhängigen verschärfte sich durch den strafrechtlich geführten »Kampf gegen die Droge«. Dennoch aber blieb die Quote der Strafaussetzungen zur Bewährung konstant bzw. sie stieg bei den Strafen zwischen einem und zwci Jahren sogar leicht an, daneben etablicrte sich die Vollstrekkungslösung. ${ }^{8}$ Strafgerichte kombinieren also selbst bei einer insgesamt repressiven Strafenpolitik die ihnen möglichen Wege der Haftvermeidung. Um Mißverständnisse zu vermeiden: Bei Drogenabhängigen empfiehlt sich eine so harte Kriminalisierung nicht, weil das Problem der Drogenabhängigen dadurch verschärft wird (vgl. Haffke, wie Fn. 4). Aber deutlich ist, daß eine Vollstreckungslösung nicht täterfreundlich gemeint ist, sondern eine harte strafrechtliche Kontrolle flankiert.

17 Thomas Fischer, Anrechnung von Drogentherapien auf Freiheitsstrafen von mehr als zwei Jahren?, SrV 1991, S. 237 lf.

I8 Monika Frommel, Therapie unter dem Druck de: Treiheitsstrate, StV 1985, S. ${ }_{889}$ ff. (Tabelle 2: Nach allgeneinem Strafreche Verurteilte mit Strafatssetzung z.ur Bewahrung). 


\section{Synopse}

\section{Rechislage bis zur Reform 1996}

S78. Sexuelle Nougung. (1) Wer einen anderen mit Gewalt oder durcDrohung mit gegeawarttger Getahr fur Lebo oder Leben notght, außerdulden oder an dem Taser oder enem Drizen vor buen an with zu Jahren bestrift (2) In munder schweren Fallen ist die Strafe Fre aaten bis zu funf Jahren.

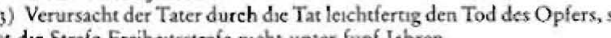

177. Vergewaltugung (1) Wer ene Frau mur Gewalt oder durch Drehung mur gegenwartuger Getabr fur Lelb oder Leben zum ausereheluche(2) In minder sehweren Fallen ist die Strafe Freheusstrafe von sechs Monaten bis zu funf Jahren. (3) Verursache der Tater durch de Tat lecchtiertig den Tod des Opfers,

179. Sexueller MiBbrauch Widerstandsuniahger. (1) Wer einen ande1 wege

wegen ener krankhaften seelischen Storung, wegen ener tefígreffen. den Bewubstsensstorung oder wegen Schwachsinns oder ciner schwe 2. korperlich widerstandsunfahig ist,

dedurch mibbraucht, daß er unter Ausnutzung der Widerstandsunfah ket auBereheliche sexuelle Handlungen an thm vornimmit oder an sich

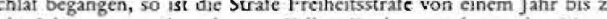
ten bis zu funf Jahren.

18.84. Begnifisbestimmungen Im Stinne dieses Gesetzes sind

nur solche, dic im Hinblick auf das ycwels geschutze Rechesgut von der Frakcionen der $\mathrm{CDU} / \mathrm{CSU}$ und F.D.P. $=$ Reformgeserz 1996 -5177
Sexuelle Nougung, Vergewaltugun

(1) Wer ene andere Person mit Gewal, durch Drohung mit gegenwarter das Opfer dem ungehemmten Einfluß des Taters pressgegeben ist, notigh sexuelle Handlungen 1. des Taxers oder

2. einer dritten Person
an sich zu dulden oder an

1. dem Tater oder

9. einer druten Perso

verzusehmen wird mit Frehheresstrafe nicht unter enem Jahr be-

straft
(2) In minder schweren Fallen use dic Straife Freehetssstrafe von sechs

(3) In besonders schweren Fallen ist die Strafe Freheitsstrafe nicht unter 2wei jahren. Ein besonders schwerer Fall liegt in der Regel vor, wena elle Hendlongen crnedron Korper verbunden sind (Vergewaltigung).

2 die Tat von mehreren gemeinschaftlich begangen wird oder 3. der Tater das Opier bel der Tat korperlich schwer miBhandelt oder es hetsschadigung bringt. (4) Verursacht der Tater durch die Tat leechefertig den Tod des Opfers, so ist die Strafe Frechentsstrate nicht unter funf Jahren

(s) Is in den Fallen des Absatzes i Nr, oder $\mathrm{Nr}$ j, auch in Verbindung kann de Tar oder Absatz 3 , das Opier mir dem Tater verheriatet, so denn, daß en besonderes offenthches Interesse an der Straiverfol gung bestcht. Der Widerspruch kann bis zur Eroffnung der Haupsverhand-

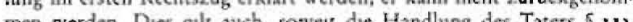
men werden. Dies gilt auch, sowent die Handlung des Taters $\{22\}$.

$$
\begin{aligned}
& \text { Sexueller Misbrauch } \\
& \text { - Misterstandsunfahtuger Personen }
\end{aligned}
$$

(1) Wer ence andere Person, dit

I. wegen einer krankhaften seelischen Storung, wegen einer teifgreffenden Bewubtsensstorung, wegen Schwachsinn oder ener schweren 2. korperhich,

zum Widerstand unfahy 1se, dadurch miBbraucht, daß er unter Ausnuezung der Widerstandsunfahigkent sexuelle Handlungen an thr vornimm oder an sich von thr vornehmen lasis, wird mit Freheitsstraite bis zu funf Jahren oder mir cldatre bestrat

(3) In besonders schweren Fallen ist die Strafe Frehhetsstrate von enem Jahr bis zu zehn Jahree. 
Gesetzentovirf
der Frakton der

\section{Sexuelle Notig}

(1) Wer etrie andere Person mut Gewalt oder durch Drohung mat gegen-

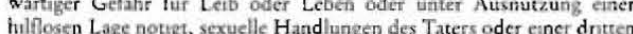
Person an sich zu dulden oder an dem Tater oder ener dritten Person vorzunehimen, wird mit Freheitsstrafe von enemen Jahr bis zu zehn Jah(2) In minds

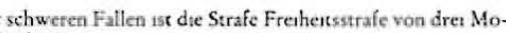

3) Verursachu der Tater durch de Tat texheferrug den Tod des Opfers, so st die Straic Frekhettsstrafe nicht unter funf Jahren

4) 5177 Abs 4 gilt enssprechenis.

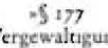

(1) Wer ene andere Person mit Gewalt oder durch Drohung mat gegenbulflosen Lage zum Beischlaf mut inmoder ener druten Person ener wird mit Frehtetsstrafe nicht unter zwel Jahten bestraf 2) In munder schweren Fallen ist die Strafe Frehlicitsstrafe von sechs Monaten bis zu funif Jahren.

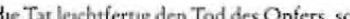
die Straie Freheressienafe necht unter funf Jahren.

Das Genchic kann die Strafe mildern oder von Strafe absechen, wemn

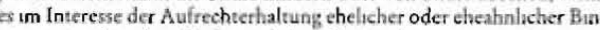

$$
\begin{aligned}
& \text { S } 179 \\
& \text { Sexueller Mifbrauch } \\
& \text { Widerstandsunfahuger }
\end{aligned}
$$

. wegen ener krankhaften seelsshen Storung, wegen ener tueftretienden Bewubssensstorung oder wegen Schwachsinns oder enner schwe. ren anderen seelischen Abartugket zum W:derstand unfahig ist odef Norperich widerstandsuntantig tst,

it sexuellc Hondlungen an thr vornunmung der Widerstandsunfahig vornehmen Laßs, wird mut Freheretsstrafe bis zu funf Jahren oder mir Geldistrafe bestratt

Wird die lat durch Misbrauch emer anderen Person zum Beschla ahren in munder schwerea Eallen Frechentsstrafe von dre I Monaten bis zu funt Jahren

(3) $\$ 177$ Abs 4 git entsprechend.

S18, c wird we folgt geander:

-(a) Als Beschiaf im Sinne deeses Abschnittes gilt auch oraler und anaier Geschlechiswerkehr
Gesetzentwionf

neten Christuna Schenk

$$
\text { Sexuelle Noti }
$$

(1) Wer ene Person gegen ihren Willen, mit Gewalt, durch Drohung mi (1) Werater ethes Dritten oder enerer Driten an sich zu dulden oder an dem Taser, sach selbst oder etinem/ener Dritten vorzunethent, wird mit Frehenstrafe nicht unter enem Jahr bestraf

(2) Wird die Tat von mehreren gemeinschafthich begangen, so ist die Straf schwere psychische oder andere schwere gesundhethche Beentrachev.

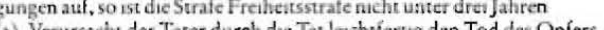
3) Verursachir der Tater durch die Tat enchtertig den Tod des Opiers,

$$
\begin{array}{rl}
5 & 177 \\
\text { vergewaltug } &
\end{array}
$$

(1) Wer eine Person gepen thren Wilien, mit Gewalt, durch Drohung mi gegenwartiger Getahr fur Letb oder Leben oder durch Drohung mite nem empinatichen Ubel zur Hinnahme anaier, oraler oder vaginaler Is-

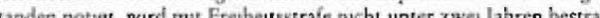
Ebenso wird bestraft, wer in glecher Weise ene Person notugt, derartuge Handlungen an sich selbst oder an emem/ einer Dritten vorzunchnen (2) Wird dec Tat von metreeren gemensischafthch begangen oder treten herer Folge beim Opjerschwere psychische oder andere schwere gesund unter dires Jahren

Tater durch die Tar lecthiterrig den Tod des Opfers. ist die Strafe Freahertsstrate nicht unter funf Jahren -

179 wird wre folgt geandert:

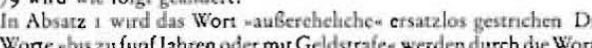

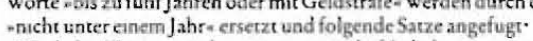

Wird dee Tat von mehreren gemensschaftuch begangen, so ist die Strafe Freeheetsstraice nucht unter zwer Jahren. Treten infolge der $\mathrm{T}$ setwere psychische oder andere sctwere gesund hetheche Beentract. Verussachr der Tater durch die Tas lacheffertio den Tod des Opfers, ist dic Strafe Freheetsstrafe nicht unter funf Jahren -

b) Absatz 2 wird wic folgt neu gefabs

(2) Wer ene Person unter Ausnutzung threr Widersandsunfahngke

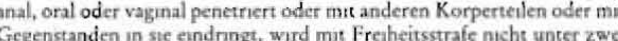
Jahren bestraft. Wiru die Tat von mehreren gemenschaftelch begangen oder treten in threr Folge bem Opfer schwer psychische oder andere schwere gesundhenthehe Beentrachtigungen aul, so ist die Strafe Free-

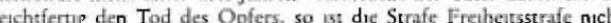
unter funf Jahren.*

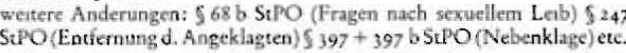

Vorschlag Frommel

Dem Einheitstatbestand des Koalrionsentwurts wird aus pragmatischen Grunden (besser als das geltende Recht) nucht widersprochen

Stat Widerspruch- oder Versohnungsklausel

ollstreckungslosurit

$$
\begin{aligned}
& 5179 \\
& \text { Sexueller Mibbrauch }
\end{aligned}
$$

(i) Wer die emgeschrankte Widerstandsfahigkecte ener anderen Person daduren mabbraucht, das er unter Aussutzung der engeschrankten W:

5) wic Koathononsentwurf

wettere Anderum

Tit-3 GVG ist dahungehend $z u$ andern, daß die Strafkamnern bern Landgencht in erster Instanz bet Straftaten nach \$ $176-179$ Verbesserungen des Opierschutzgesetzes 